\title{
¿CUÁL BRILLA MÁS?: PREDICCIONES Y REFLEXIONES ACERCA DEL BRILLO DE LAS BOMBILLAS
}

\author{
SEBASTIÁ, J.M. \\ Departamento de Física. Universidad Simón Bolívar. Caracas. Venezuela.
}

\section{SUMMARY}

This work analyses the extension, consistency and stability of university students' interpretations concerning electric circuits. We also develope some theoretical considerations about the origin of the students' interpretations and their possibilities of transformation and change.

\section{INTRODUCCIÓN}

Voltaje, tensión, corriente, resistencia, etc. son términos de un lenguaje común con el que todos estamos familiarizados al utilizarlos para referirnos al funcionamiento de una gran cantidad de aparatos eléctricos y electrónicos de nuestro entorno cotidiano.

Las ideas básicas de electricidad son presentadas a Ios estudiantes en los cursos de física del bachillerato, frecuentemente acompañadas de experimentos sencillos con baterías y bombillas. Como consecuencia, muchos estudiantes de bachillerato, y todavía más los de universidad, son capaces, a veces, de resolver complicados circuitos con mallas eléctricas y de realizar montajes eléctricos relativamente sofisticados.

Parecería pues, a primera vista, que la electricidad es una đe las áreas más atractivas y menos problemáticas de los cursos de física. Sin embargo, Ia realidad es otra. A pesar de la destreza que exhiben los estudiantes en el manejo de las ecuaciones y en los montajes experimentales, se ha hecho patente que los estudiantes interpretan los circuitos eléctricos de una manera que dista mucho de ser la que se pretende transmitir en las clases de Física (Cohen, Eylon y Ganiel 1983, Osborne 1983, Closset 1983). Estas interpretaciones se manifiestan, preferentemente, cuando los estudiantes no pueden refugiarse en el manejo de fórmulas o de expresiones memorizadas.

La extensión y fortaleza de algunas de estas ideas, o conceptualizaciones alternativas, han sido exploradas por diferentes investigadores (Danusso y Dupré 1987 , Buchweitz y Moreira 1987, Dupin y Johsua 1987, Gauld 1988, Varela, Manrique y Favières 1988, Acevedo 1989 , Andrés 1990, Licht y Thijs 1990). Las principales características de las interpretaciones de los estudiantes sobre los circuitos eléctricos que han destacado los investigadores son:

a) La idea de que Ia bombilla conslime o atenúa la corriente que circula por ella. Cuando se colocan varias bombillas en serie, los estudiantes predicen que la bombilla más cercana al punto đe donde «\$ale» la corriente brillará con mayor intensidad que las otras, ya que la corriente se va «gastando» al atravesar Ias sucesivas bombillas.

b) La idea de que la corriente produce voltaje. Una interrupción de la corriente, como la que se produce al abrir un circuito, conlleva, para muchos estudiantes, la desaparición del voltaje entre cualquiera de los dos puntos del circuito.

c) Un tipo de razonamiento secuencial para analizar eI circuito. Si el circuito se modifica en un punto, por ejemplo aumentando su resistencia, sólo se verían afectados, según este razonamiento, los elementos que se encuentran «corriente abajo» en el circuito, y no los que ya recorrió la corriente.

Nuestra investigación no se proponía como interés principal la amplicación de la ya abundante base de datos de 
las concepciones alternativas en electricidad y circuitos, más bien estaba orientada a obtener resultados que pudieran enriquecer la điscusión teórica sobre los aspectos relacionados con el origen y posibilidades de cambios de estas concepciones. Con este fin se elaboró un cuestionario que permitiera analizar la consistencia y estabilidad de las interpretaciones de los estudiantes y se aplicó a una muestra de estudiantes universitarios; los resultados obtenidos, aunque ya venían precedidos por abundante investigación en el área, no dejaron de ser sorprendentes.

\section{METODOLOGIA: MUESTRA E INSTRU- MENTO}

El diseño experimental consistió en un estudio transseccional de una muestra estratificada en función de su nivel de avance en las asignaturas de Física Básica y Redes Eléctricas (lo cual pretendia ser un indicador de la familiaridad de los estudiantes con los circuitos eléctricos). La muestra estaba constituida por 273 estudiantes de la Universidad Simón Bolívar de Caracas (Venezuela). Algunos datos característicos de la muestra son los que aparecen en la tabla $I$.

Tabla I

Caracterización de la muestra.

\begin{tabular}{|l|l|c|c|}
\hline Estrato & Nivel educativo & N. de sujetos & Edad (años) \\
\hline E1 & Primer año (Física 1) & 47 & 17,6 \\
\hline E2 & Segundo año (Física 3) & 63 & 19,4 \\
\hline E3 & Segundo año (Redes 1) & 110 & 19,7 \\
\hline E4 & Tercer año (Redes 4) & 53 & 20.8 \\
\hline
\end{tabular}

El estrato EI estaba constituido por 47 estudiantes de la asignatura de Física 1, pertenecientes a las carreras de Ciencias e Ingeniería que ofrece la Universidad; únicamente habían cursado física en el bachillerato y se estaban iniciando en la física universitaria. El estrato E2 lo constituían 63 estudiantes de la asignatura de Física 3, que ya habían seguido dos cursos universitarios de física, con contenidos de mecánica y termodinámica, pero no habían cursado circuitos eléctricos a nivel universitario.

El estrato E3 estaba constituido por 110 estudiantes de la asignatura de Redes Eléctricas 1, de las carreras de ingeniería eléctrica e ingeniería electrónica, los cuales habían estudiado circuitos eléctricos dentro de los cursos de f́́sica básica. El estrato E4 lo constituían 53 estudiantes de la asignatura de Redes Eléctricas 4, con una alta especialización en circuitos.

La razón fundamental de haber escogido esta estratificación de la muestra era la de analizar la influencia que la formación especializada tenía sobre las diferentes interpretaciones altemativas que realizan los estudiantes acerca de los circuitos eléctricos. Observar cuáles eran fácilmente removibles y cuáles se resistían a ser alteradas, aspectos centrales a la teorización acerca de la dinámica conceptual.

El instrumento utilizado estaba constituido por un test integrado por ocho situaciones de circuitos eléctricos elementales con baterías, bombillas y resistencias. La mayoría de las situaciones eran adaptaciones de las formuladas por Dupin y Johsua (1987) y Shipstone y otros (1988); estaban diseñadas en forma de selección múltiple y la opción a escoger debía ser complementada con explicaciones o justificaciones escritas del estudiante.

\section{ALGUNOS RESULTADOS (;SORPRENDEN- TES!)}

Aunque las ideas que poseen los estudiantes no son accesibles de manera directa, éstas conducen a predicciones concretas, las cuales pueden ser confirmadas o desconfirmadas por la experiencia.

La predicción más evidente referida a los circuitos eléctricos es la concerniente al brillo de las bombillas que componen el circuito. La respuesta a la cuestión: «Dadas dos o más bombillas en el circuito contestar a la pregunta: ¿Cuál brilla más?» puede «arrojar luz» acerca de la manera en la que el estudiante está concibiendo el funcionamiento eléctrico del circuito y acerca de cómo concibe el papel que juegan en dicho funcionamiento los elementos que lo componen.

Por ejemplo, dado el circuito eléctrico de la figura 1 , ante la pregunta ¿cuál brilla más?, Ios estudiantes que conciben el circuito como una serie de elementos interactuantes, que influyen conjuntamente en la intensidad de corriente que proporciona la batería, responderán que «ambas bombillas, L1 y L2, brillan igual», to cual es correcto.

Por el contrario, aquéllos que conciben el circuito de manera secuencial, paso a paso, interpretan, incorrectamente, que la resistencia consume parte de la corriente que circula por ella, por lo tanto, si la resistencia está colocada antes de la bombilla disminuirá la intensidad de corriente que le llega a ésta, y por lo tanto disminuirá el brillo de la bombilla. En este caso responderán «que la bombilla $\mathrm{L} 2$ brillará más que la bombilla $\mathrm{L} 1 »$.

Sorprendentemente, no llegó a alcanzar la proporción de un tercio $(29,8 \%)$ de los estudiantes de primer año universitario los que seleccionaron la opción correcta: «ambas brillan igual». Los otros dos tercios $(68,1 \%)$ consideraron más apropiada Ja opción incorrecta: «L2 brilla más que L1», haciendo patente la popularidad del razonamiento espontáneo mencionado al comienzo de este artículo.

La figura 2 muestra otra situación de un circuito elemental en el cual se modifica el valor de una de las resistencias: ¿Qué sucederá con el brillo de las bombillas si R2 se duplica? Concebido el circuito como un sistema, el aumento de una resistencia conducirá a una disminución 
Figura 1

En el circuito de la figura, las resistencias y las bombillas son idénticas. ¿Cuál de las siguientes afirmaciones crees que es correcta?
a) L2 brilla menos que Ll.
b) L2 brilla igual que L1.
c) L2 brilla más que L1.

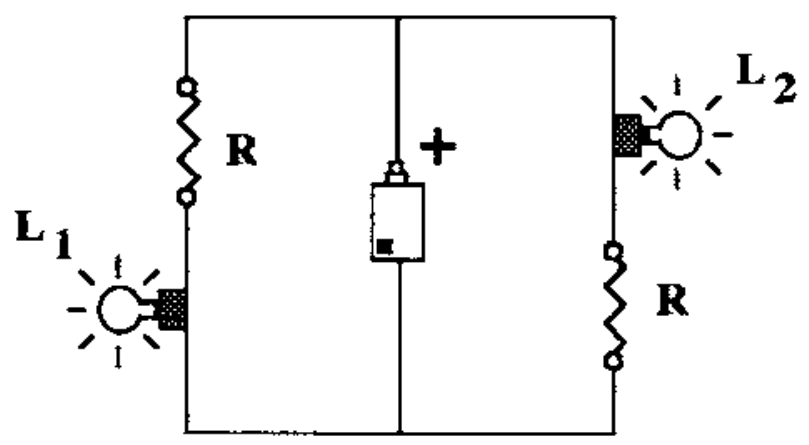

de la intensidad de corriente que proporcionará la batería y por lo tanto a una disminución del brillo de ambas bombillas. Por el contrario, concebido en el circuito, incorrectamente, de manera secuencial, el aumento del valor de la resistencia $\mathrm{R} 2$ conduciría a una disminución del brillo de la bombilla L2, pero dejaría inalterado el brillo de la bombilla Li.

Figura 2

En el circuito de la figura, las bombillas son idénticas. Inicialmente las resistencias $\mathrm{Rl}$ y $\mathrm{R} 2$ son iguales. Si el valor de la resistencia R2 se duplica, podemos afirmar que:

a) El brillo de $\mathrm{L} 1$ y $\mathrm{L} 2$ permanece igual.

b) El brillo de L2 disminuye y el brillo de Ll permanece igual.

c)El brillo de L1 disminuye y el de L2 permanece igual.

d) El brillo de L1 y L2 disminuye por igual.

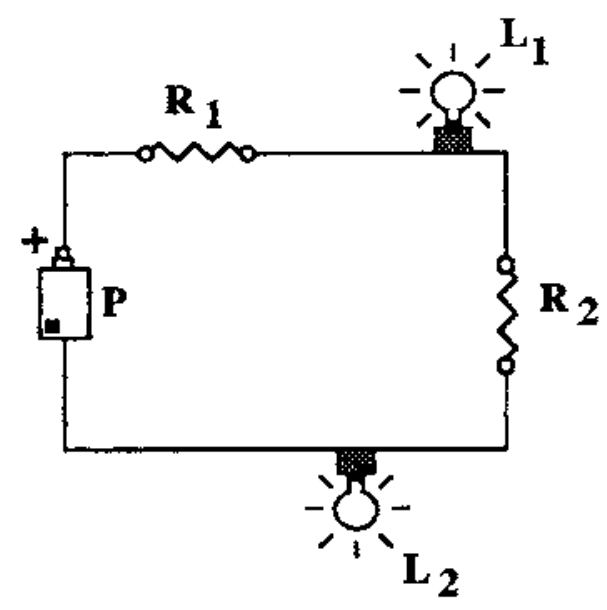

Los resultados reflejan que no llegan a la quinta parte $(19,1 \%)$ los estudiantes universitarios de primer año entrevistados que respondieron correctamente: «El brillo de ambas bombillas disminuye por igual». Dos tercios $(65,9 \%)$ de este estrato de la muestra escogió la opción que reflejaba un razonamiento secuencial: «El brillo de L 2 disminuye y el de LI queda igual». Un tercer ejemplo, de los contemplados en el instrumento, lo constituye la situación presentada en la figura $3^{1}$.

La situación presenta dos circuitos esquematizados: el circuito $a$ con una sola bombilla y el circuito $b$ con dos bombillas en paralelo. La pregunta que se formulaba a los estudiantes era Ia clásica: «Cuál brilla más?». La respuesta correcta, basada en que las tres bombillas están conectadas a una misma diferencia de potencial (voltaje) de baterías idénticas, sería: «Todas brillan igual». Otra manera, tambien correcta, de razonar sería observar que la resistencia equivalente del circuito $b$ es la mitad del circuito $a$, por lo tanto la batería proporciona en el caso $b$ el doble de corriente que $a$ y al ser dividida en partes iguales, las bombillas L 2 y L 3 serán atravesadas por la misma intensidad de corriente que LI y por lo tanto brillarán igual.

Figura 3

En los circuitos que se indican a continuación, las pilas y las bombillas son idénticas. En relación con el brillo de las bombillas podemos afirmar que:

a) $\mathrm{S} 6 \mathrm{l}_{\mathrm{O}} \mathrm{L}_{3}$ y $\mathrm{L}_{2}$ brillan igual.

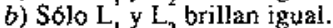

c) $L_{3}$ y $L_{3}$ brillan menos que $L_{1}$

d) Todas brilian gitual.
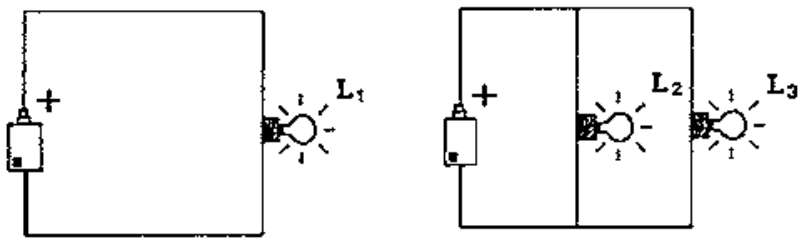

Por el contrario, los estudiantes que razonen que en el circuito $b$ la batería tiene que alimentar a dos bombillas mientras en el circuito $a$ tenía que hacerlo a una sola, sin tener en cuenta que esto afecta a la intensidad de corriente que genera la batería, concluirán erronéamente, que «las bombillas L2 y L3 brillan menos que L1».

De nuevo, poco más de un tercio $(38,3 \%)$ de los estudiantes de primer año universitario seleccionó la opción correcta. Casi dos tercios $(55,3 \%)$ se inclinaron por la opción alternativa.

Ahora bien, ¿cómo evolucionan estas interpretaciones con la enseñanza formal de los estudiantes? Sorprendentemente, de nuevo, la evolución de las interpretaciones que realizaron estudiantes fue sumamente dispar. La figura 4 expone la evolución de los porcentajes de respuesta correcta a las tres situaciones presentadas anteriomente.

La proporción de respuestas correctas al f́tem que muestra la figura 1 experimenta una variación casi lineal $(29,8 \%$ en E1, $41,3 \%$ en E2, $59,1 \%$ en E3 y $100 \%$ en E4). Únicamente sorprende el hecho de que no supera el $60 \%$ de los estudiantes de las carreras de ingeniería eléctrica y electrónica que son capaces de hacer una interpretación correcta e nivel de un segundo año de carrera. 
Figura 4

\section{ESTRATO}

Evolución de las respuestas correctas a las situaciones de los circuitos de las figuras 1,2 y 3.

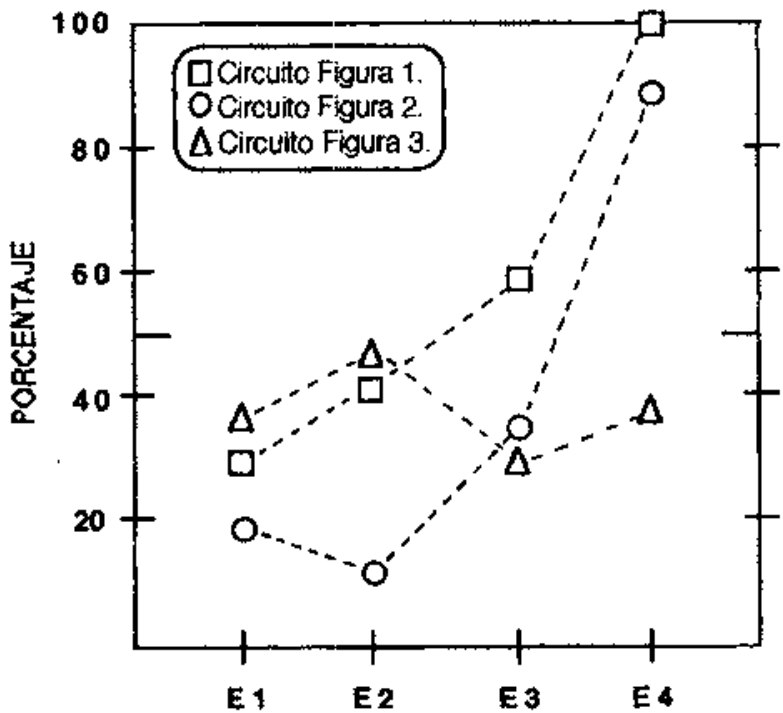

Los porcentajes de respuesta correcta a la situación mostrada en la figura 2 tienen un patrón de evolución diferente. Comienza con un valor sumamente bajo $(19,1 \%$ en $\mathrm{El}$ ), disminuye al aumentar el nivel educativo (15,9\% en E2), aumenta notablemente, aunque dentro de valores relativamente bajos en el siguiente estrato $(37,7 \%$ en E3), y aumenta significativamente con la especialización en redes eléctricas ( $92,4 \%$ en E4). La regresión que experimenta el porcentaje de respuestas correctas con el nivel educativo entre los dos primeros estratos da lugar a to que algunos han Ilamado evolución en $U$ de las respuestas de los estudiantes (U - Shaped Behavior, Happs 1985).

Pero, sin duda, el resultado más destacado corresponde al de la situación mostrada en la figura 3. La proporción de respuestas correctas permanece prácticamente estancada en un entorno del $40 \%(38,3 \%$ en el El, $42,9 \%$ en $\mathrm{E} 2,37,3 \%$ en E3 y $39,6 \%$ en E4). Aunque las justificaciones de los estudiantes se hacen más sofisticadas al avanzar en su formación académica, la esencia de su interpretación permanece inalterada. Incluso los estudiantes que están culminando su formación en redes eléctricas responden inadecuadamente a esta pregunta

\section{ALGUNAS REFLEXIONES ACERCA DE LOS RESULTADOS}

\section{¿De dónde provienen las ideas espontáneas?}

Si aceptamos la premisa constructivista de que el estudiante no llega a la situación de enseñanza con la mente en blanco, sino que ya posee ciertas iđeas espontáneas o conceptos previos que mediatizan sus interpretaciones de la realidad, surge un interrogante evidente: ¿Cuál es el origen de dichas ideas?

Frente a este interrogante, los investigadores han adoptado distintas posiciones. Algunos han destacado la importancia que los factores externos al individuo tienen en la generación de estas ideas. Solomon y Black (1987), por ejemplo, han rastreado el origen de Ias ideas de electricidad y energía inducidas por el contexto social y transmitidas a través del lenguaje. McCloskey (1983), entre otros, ha resaltado el origen perceptivo o empírico de muchas nociones que manejan los estudiantes, en particular en las interpretaciones del movimiento, posiblemente unido a generalizaciones superficiales de las mismas (Gil y Carrascosa 1990).

Castro y Fernández (1989), siguiendo la línea piagetiaaa, atribuyen el origen de dichas ideas a una inadecuada adaptación de la realidad por el sujeto, debido supuestamente a que el estudiante no ha alcanzado niveles formales o incluso postformales de conocimiento.

Desde una perspectiva racionalista, Preece (1984) y Sebastiá (1989) han retomado las propuestas que Fodor (1975) y Chomsky (1980) desarrollaron para interpretar la similitud de estructuras linguísticas en diferentes culturas, sugiriendo la existencia de ciertos «a priori» cognitivos que canalizan la interpretación de la realidad en una cierta forma, conduciendo a los estudiantes a realizar interpretaciones «naturales» similares, al tiempo que obtaculizan otras interpretaciones «no naturales\%.

Ahora bien, ¿qué aportan los resultados obtenidos en esta investigación al debate sobre el origen de las interpretaciones espontáneas?

Las ideas de los estudiantes constituyen un heterogéneo conglomerado cuyo origen es difícil de rastrear. La idea de que los elementos del circuito (resistencias o bombillas) consumen electricidad, según evidencian nuestros resultados, junto con los de casi todos los anteriores investigadores está en la raíz de muchas de las predicciones erróneas de los estudiantes. A esta idea se le puede atribuir un origen empírico, ya que es un hecho experimental conocido por todos que la electricidad produce «Juz y calor» en las bombillas, lo cual puede ser interpretado por el estudiante como una «transformación» de la electricidad en «luz y calor».

Por otro lado, la idea de producción de electricidad en las baterías y consumo en las bombillas concuerda con un esquema de razonamiento causal-iineal que ya ha sido explorado en otros campos y que conduce, en ocasiones, a predicciones correctas (Andersson 1986, Sebastiá 1989). En los circuitos-serie, la atribución de una relación causa-efecto a la batería y al brillo de las bombillas no conduce a contradicciones experimentales. Sin embargo, los circuitos-paralelos no pueden ser analizados mediante este esquema, ya que, por ejemplo, si aumenta el número de baterías en paralelo, esto no conlleva un aumento del brillo de las bombillas del circuito. 
Así pues, los resultados obtenidos abundan en la importancia de la idea de consumo de corriente en las bombillas como idea clave para generar predicciones incorrectas, particularmente en circtitos-paralelo. Ahora bien, esta idea aparece en la confluencia, y quizas por esa razón es tan difícil de eliminar, de las generaciones empíricas superficiales (Gil y Carrascosa 1990) y de las atribuciones causales (Andersson 1986).

\section{¿Cambio conceptual o comprensión conceptual?}

La dinámica đel conocimiento humano es un asunto complejo. No consiste simplemente en añadir datos a la memoria, requiere frecuentemente evaluar la nueva información, compararla con la ya existente y posiblemente descartar algunas de las creencias que ya teníamos (Gärdenfors 1990).

En la dinámica del conocimiento humano, el cambio conceptual es entendido (White y Gunstone 1989) como la aceptación de un nuevo esquema conceptual acerca de un determinado fenómeno y el abandono del anterior.

Aunque algunas ideas resultan fáciles de cambiar, existe una clase de ideas, esquemas o relaciones que se manifiestan tenazmente resistentes a ser suplantadas. Por ejemplo, la asignación de fuerza al movimiento, la vinculación calor-temperatura o, en este caso, el razonamiento secuencial en circuitos eléctricos.

Estas ideas reaparecen incluso cuando ya se las creía eliminadas. Al parecer, siempre vuelven a aflorar las nociones anteriores, según han evidenciado diferentes investigaciones (Happs 1985, Closset 1989, Viennot 1989).

La perspectiva del cambio conceptual está basada en la hipótesis de que no pueden coexistir, en una misma persona, dos esquemas de conocimiento mutuamente incompatibles. Ahora bien, ¿es ésta una suposición fundamentada? Algunos autores (Castro y Fernández 1987 , Licht 1987, Tiberghien 1989, Johsua 1989, Sebastiá 1989) han sugerido que es perfectamente factible que en un mismo sujeto coexistan concepciones aparentemente contradictorias pero que son utilizadas especificamente según el contexto. Es decir, los diferentes tipos de conocimiento serían codificados de distinta manera, responderían a exigencias epistemológicas diferentes y serían accesibles y utilizables atendiendo a sus campos de práctica particulares.

Si es posible, y al parecer inevitable, que el individuo comparta simúltaneamente más de un esquema conceptual acerca de un contenido determinado, ¿qué implicaciones conlleva esto para la enseñanza de la ciencia? La principal consecuencia apunta a que la estrategia de cambio conceptual debe ser revisada. Aprender ciencia, desde esta nueva perspectiva, no consiste en suplantar unos conocimientos por otros, sino más bien, en aprender a distingir los contextos en los que son aplicables los diferentes esquemas conceptuales.
¿Qué aportan nuestros resultados a esta polémica del cambio conceptual? La evolución de la proporción de respuestas correctas manifiesta poca variación entre los primeros niveles $(p<0,1)$ y elevada variación entre los niveles de alta especialización ( $p<0,001)$. En casi todas las situaciones se alcanza un cambio de esquema predictivo-interpretativo notable ¿Podría concluirse que Ia enseñanza ha logrado propiciar el cambio conceptual en el estudiante? Para poder concluir que se ha producido un cambio conceptual se requeriría que, cuando el estudiante ha reemplazado un esquema para analizar una situación, todas las situaciones afines sean también interpretadas con el nuevo esquema. Esto implicaría que debería aparecer una alta correlación entre los resultados a situaciones afines; sin embargo, los resultados de correlación arrojan cifras relativamente bajas.

La excepcional estabilidad de algunas situaciones, como la referida a la situación de la figura 3 , así como la poca correlación entre interpretaciones de situaciones análogas hacen pensar que, como ha sugerido Di Sessa (1985), los estudiantes interpretan la realidad en base a fragmentos de conocimiento poco interconectados, más bien que haciendo uso de teorías. Las variantes de una determinada situación son frecuentemente interpretadas por su analogía con la precedente, pero, cuando el estudiante no identifica la nueva situación como una variante de la anterior, regresa al esquema interpretativo que aparentemente había abandonado. Desde esta perspectiva, el conocimiento precedente no es eliminado, sino que permanece latente, los diferentes conocimientos son relacionados con diferentes «dominios de práctica» (Tiberghien 1989) y pueden reaparecer cuando la situación parezca requerirlo.

\section{CONCLUSIONES}

En el presente trabajo hemos presentado algunos resultados obtenidos mediante un estudio «trans-seccional» acerca de la comprensión de los circuitos eléctricos en estudiantes universitarios.

La indagación tenía fundamentalmente el objetivo de enriquecer la discusión teórica acerca del origen y el cambio de las concepciones alternativas o espontáneas de los estudiantes. Ambos aspectos son complejos y en ninguno de ellos era de esperar una respuesta sencilla.

Con relación al origen de las interpretaciones alternativas de los circuitos eléctricos, los resultados permiten múltiples interpretaciones. En este artículo han sido analizadas algunas de ellas en función de su compatibilidad con los resultados obtenidos.

Con respecto a la disyuntiva entre estabilidad y cambio conceptual, los resultados apuntan en la dirección de que un cambio conceptual, entendido como el reemplazo global de unas ideas por otras, no se produce en la mente del estudiante. Lo que parece ocurrir es un avance de «fragmentos de conocimiento», que nunca llegan a sus- 
plantar totalmente a los anteriores; estos «fragmentos de conocimiento» ligados a «prototipos» específicos permiten entender la disparidad de la evolución de las respuestás y el porqué se recurre a conocimientos ante. riores cuando las situaciones no son identificadas como variantes del «prototipo» aceptado.

\section{REFERENCIAS BIBLIOGRÁFICAS}

ACEVEDO, J.A., 1989. Las interpretaciones de los estudiantcs de BUP sobre electrocinética, Investigación en la Escuela, 7, pp. 107-115.

ANDERSSON, B.R., 1986. The experimental gestalt of causation: A common core to pupils preconceptions in science, European Journal of Science Education, Vol. 8, pp. 155-171.

ANDRÉS, M.M., 1990. Evaluación de un plan instruccional dirigido hacia la evolución de las concepciones de los estudiantes acerca de circuitos eléctricos, Enseñanza de las Ciencias, Vol. 8(3), pp. 231-237.

BUCHWEITZ, B. y MOREIRA, M.A., 1987. Misconceptions in physics: research findings among Brazilian students. Second International Seminar on Misconceptions and Educational Strategies in Science and Mathematics. Cornell University. (Ithaca: Nueva York).

CASTRO, E.A. y FERNÁNDEZ, F.M. Intellectual development beyond formal operations, International Journal of Science Education, Vol. 9(4), pp. 441-447.

CLOSSE'T, J.L., 1983. Sequential reasoning in electricity, en «Research on Physics Education». Proceedings of the First International Workshop. (La Londe: Les Maures), pp. 313319

CLOSSET, J.L., 1989. Les Obstacles à l'apprentissage de l'électrocinétique, Bulletin de L'Union des physiciens, 716 , pp. 913-949.

CHOMSKY, N., 1980. Rules and representations. (Columbia University Press: Nueva York).

DANUSSO, L. y DUPRE, F., 1987. Student representation of simple electric circuits. Second International Seminar on Misconceptions and Educational Strategies in Science and Mathematics. (Cornell University. Ithaca: Nueva York).

DI SESSA, A.A., 1985. Knowledge in pieces. (University of California: Berkeley).

DUPIN, J.J. y JOHSUA, S., 1987. Conceptions of French pupiis concerning electric circuits: structure and evolution, Journal of Research in Science Teaching, Vol. 24(9), pp. 791-806.

FODOR, J.A., 1975. The language of Thought. (Thomas Y. Crowell: Nueva York).

GÄRDENFORS, P., 1990. The dynamics of belief systems: foundations versus coherence theories, Revue Internationale de Philosophie, Vol, 44(172), pp. 24-46.

GIL, D. y CARRASCOSA, T., 1990. What to do about science «Misconceptions»?, Science Education, Vol. 74(5), pp. $531-540$.

\section{NOTA}

1 Una información completa acerca del instrumento utilizado, resultados obtenidos y análisis de correlación realizados puede ser solicitada directamente al autor.

HAPPS, J.C., 1985. Regression in learning outcomes: some examples from the earth sciences, European Journal of Science Education, Vol. 7, pp. 431-443.

LICHT, P., 1987. Strategy to deal with conceptual and reasoning problems in introductory electricity education. Second International Seminar on Misconceptions and Educational Strategies in Science and Mathematics. (Cornell University. Ithaca: Nueva York).

LICHT, P. y THIJS, G.D., 1990. Method to trace coherence and persistence of preconceptions, International Journal of Science Education, Vol. 12(4), pp. 403-416.

MCCLOSKEY, M., 1983. Intuitive Physics, Scientific American, Vol. 248, pp. 122-130.

OSBORNE, R., 1983. Towards modifying children's ideas about electric current, Research in Science and Technological Education, Vol. 1(1), pp. 73-82.

PREECE, P.F.W., 1984. Intuitive science: learned or triggered?, European Journal of Science Education, Vol. 6(1), pp. $7-10$

SEBASTIÁ, J.M., 1989. Cognitive constraints and spontaneous interpretations in physics, International Journal of Science Education, Vol. Il(4), pp. 363-369.

SHIPSTONE, D.M. et al., 1988. Study of students understanding of electricity in five European countries, International Journal of Science Education, Vol. 10(3), pp. 303-316.

SOLOMON, J. y BLACK, P., 1987. The pupil's view of electricity revisited: social development or cognitive growth?, International Journal of Science Education, Vol. 9(1), pp. 13-22.

TIBERGHIEN, A., 1989. Phénomenés et situations matérielles: quelles interpretetions pour l'élève et pour te physicien? Constructions des Savoirs: obstacles \& conflits. (Les Editions Agence D'Arc: Ottawa).

VALERA, P., MANRIQUE, M.J. y FAVIËRES, A., 1988. Circuitos eléctricos: una aplicación de un modelo de enseñanzaaprendizaje basado en las ideas previas de los alumnos, Enseñanza de las Ciencias, Vol. 6(3), pp. 285-290.

VIENNOT,L., 1989. Bilans de forces et loi des actions réciproques. Analyse des difficultés des élèves et enjeux didactiques, Bulletin de L'Union des Physciens, (716), pp. 951-971.

WHITE, R.T. y GUNSTONE, R.T., 1987. Metalearning and conceptual change, International Journal of Science Education, Vol. 11, pp. $577-586$ 